\section{Change of Address}

The Association for Research in Infant and Child Development (formerly of London N.W.3) has changed its address to: 4 North Drive, Great Baddow, Chelmsford, Essex.

\section{COMING EVENTS}

Royal Northern Hospital Postgraduate Centre, London.-Details of postgraduate teaching sessions, Mondays and Fridays, 2-27 August, are obtainable from the dean's secretary, Royal Northern Hospital, London N7 6LD. (Tel. 01-272 7777, (extn. 218.)

"Control of Human Ageing."-International study forum, 2-4 September, near Zurich, organized by the Gottlieb Duttweiler Institute for Economic and Social Studies. Fee Swiss francs 685. Details from the congress secretariat, G. D. Institute, CH-8803 Rüschlikon-Zurich, Switzerland.

4th European Symposium on Epilepsy.6-8 September, Amsterdam. Details from the symposium secretariat, c/o Municipal Congress Bureau, 199 O.Z. Achterburgwal, Amsterdam-C, Netherlands.

Computers for Analysis and Control in Medical and Biological Research.-Conference, 7-9 September, Sheffield University. Details from the Manager, Conference Department, Institution of Electrical Engineers, Savoy Place, London WC2R OBL.

16th Congress of Paediatrics.-With international participation, 9-11 September, Prague. Details from the secretary general of the Congress, Sokolská 31, Prague 2, Czechoslovakia.

"Human Locomotor Engineering."-Conference by the Institutions of Mechanical Engineers and of Electrical Engineers, 7-10 September, Sussex University. Registration forms and details from the Institution of Mechanical Engineers, 1 Birdcage Walk, Westminster, London S.W.1.

8th British Congress on the History of Medicine.-8-11 September, Liverpool. Details from the secretary of the congress, Wellcome Institute of the History of Medicine, 183 Euston Road, London N.W.1.

Institute of Orthopaedics.-Instructional course, "Infective and Parasitic Disorders," Wednesdays, 15 September-15 December. Details of this and other courses are obtainable from the secretary for postgraduate studies, at the institute, Roya National Orthopaedic Hospital, 234 Great Portland Street, London W1N 6AD. (Tel. 01-387 5070.)

\section{APPOINTMENTS}

United Birmingham Hospitals.-P. Hicken, M.D. D.M.R.D. (consultant radiologist)

South-EAST MEtropolitan Regional Hospital BOARD.-M. C. Bott, M.B., D.P.M. (consultant, psychiatry, Hailsham and Hastings groups); A. D. C Norris, M.B., M.R.C.P. (consultant, oeneral mediM.Phil., M.R.C.P. (consultant, psychiatry (child guidance), Brighton and Lewes, and St. Francis and the Lady Chichester groups); f. M. Howat. M.B., and Lewes group); J. S. R. Baxter, M.B., F.R.C.S (consultant, E.N.T., Canterbury and Isle of Thane groups); M S. Davies, M.B., F.R.C..S., D.O. (con sultant, ophthalmology, Medway and Gravesend, and Central Kent groups); Rachel G. Evans, M.B.,
M.R.C.P., D.C.H. (consultant, paediatrics, Lewisham, M.R.C.P., D.C.H. (consultant, paediatrics, Lewisham, and Greenwich and Deptford groups); F. S thetics, Hastings group); F. E. Bennetts, M.B. thetics, Hastings group); F. E. B.C.S. (consultant, anaesthetics, Central Kent, Medway and Gravesend groups); J. A. Hatrick, Darenth and Stone group); B. E. Crawley, M.B. F.F.A. R.C.S consultant, anaesthetics, Canterbury and Isle of Thanet groups)

\section{UNIVERSITIES AND COLLEGES}

\section{CAMBRIDGE}

M.D.-G. S. Panayi.
M.CHIR.-R. G. Pulvertaft, W. H. Davies.
M.B.-R. J. G. Rycroft.

ST. ANDREWS

On 2 July the following degrees were conferred

M.B., CH.B.-1J. E. H. Brice, 1A. J. Moulds,
1Anne M. Worthington, 1Anne P. Dunbar, 1Jennifer M. Hunter, D. J. M. Allan, Janice B. Beveridge, S. M Booker, M. A. Briggs, Anne D. Brimley, G. G. P. Browning, M. J. T. Carr, G. A. Carter, R. Clark,
I. D. Conacher, M. D. Cowan, B. J. S. Craig, Helen
J. C. Crowe, B. C. Dawe, J. A. Denner, II. Dewhurst, Sheena M. Douglas, I. G. Drummond, Forrester, K. M. Fox, G. P. Hadley, Kathleen M. A. H. Hornsby, J. C. Islip, J. D. Lewis, R. M. A. H. Hornst, J.i C. Islip, J. D. Lewis, R. M.
D. Mackay, Jennifer J. McNab, I. S. McNeill, W. A. DacRae, Mennifer J. McNab, I. S. McNeill, W. A. Mohamdee, M. B. S. Ramadam, R. H. Robertson, Christine E. Ross, E. R. S. Ross, M. J. W. Scott Soukop, G. J. Stewart, R. Talbot-Jones, G. E. Taylor, N. C. Taylor, B. D. Walker, A. E. Wallin
R. J. Wherton, D. M. Whitcher, A. F. Wright.

\section{ABERDEEN}

At recent ceremonies the honorary degree of Doctor of Laws was conferred on the following

Dr. J. H. F. Brotherston, professor of public health and social medicine in Edinburgh University, 1955-64; chief medical officer, Scottish Home and
Health Department since 1964. Sir Stanley Davidson, regius professor of medicine in Aberdeen University, 1930-8; profe Dr. Alexander Lyall, lecturer in clinical chemistry
and head of that department, Aberdeen University, 1926-61; general council assessor to the university M.D.-M. McA. Lees (with commendation), T. Ashcroft. In the Faculty of Medicine: S. W. B. Mw.B., Cн.B.-T. A. Akintewe, B. G. Anderson, Christobel G. Bahzad, J. L. Baird, A. B. Barber,
J. E. Barclay, Allexandra M. Barrett-Ayres, A. G.
Beattie, W. R. A. Beattie, Alice M. Bisset. S. G. Boyd, M. J. Buchan, Helen J. Carr. Sheila E. Carr. B. J. Carrie Christopher M. Childs, Dawn E.
Douglas, Lydia F. Fell, M. M. A. Dean, G. A. Finlayson, Aileen F. Fleming, Anne Forster, D. R Garrioch, E. H. Gomes, Joan L. Groodall, P. J.
Gordon, H. S. Grant, J. A. Grant, Sheila B. Grant,
Janet M. Henderson, P. R. Henderson, I. K. C. Hunter, A. A. Hutchison, Patricia A. Inoram, W. I. Ingram, R. A. Hutchison, Patricia A. Inoram, W. T. Ingram, R. A. Keenan, J. F. Kinnon, Helen E. L indsay, R. J. Lydon, R. J. MacP. MacDonald,
Christina E. McKay, P. N. G. Mackay A. G McKee, A. McNeill, J. M. Mair. Z. G. Mamujee, Abdul-Marid A. Masood, Helen W. Massie. M. I. Metcalf, G. W. Milne, Eivind Molven, Jean I. Pearson, G. R. Petrie, S. Photinu. J. P. Reid,
I. A. N. Rennie, Marraret C. G. Richardson, A. B.
Roberts. Helen C. Robertson, G. D. Rose. J. S Roberts. Helen C. Robertson, G. D. Rose, J. S. Cosie, M. F. Shanks, A. F. Shrarer, A. I. Shearer, D. W. Sinclair, D. J. M. Sinclair, J. N. Slater, Wilson. Judith A. Barnabv, G. H. Barnes, Dorothy W. B:rks, C. C. Scott, J. Sue-Chue-Lam, A. D. Thomson.
Dip. PsychotheraPY.-Máire O'Shea, Jin-Inn
Teok. NEWCASTLE UPON TYNE

Annointments.-Dr. J. A. Barrowman (reader in (lecturer, department of pathology).

WALES

M.D.-T. G. Davies, A. D. Griffiths, R. E. Jones, J. E. Morris. H. Davies, Shirley W. Hampson, T. T. Jones, Esther H. Rees, Margaret M. S
Sibani Sarkar, R. S. Vaid, T. T. Westhead.

QUEEN'S UNIVERSITY OF BELFAST

Appointments.-Dr. F. V. O'Brien (lecturer, dental
patholooy); Dr. A. patholoqy); Dr. A. H. Nagi (lecturer, pathology) pharmacology).

DUNDEE

On 12 July the following degrees were conferred

HoN. LL.D.-Dr. Eliot T. O. Slater.

CH.M.-1j. C. Forrester

M.B., CH.B.-Eileen M. C. Duke, Angela A. S. Bowring, R. W. Gourdie, Moira E. V. McKiddie, Kathleen Martin, Elizabeth Mowat,
McQ. E. Orr, M. Rogers, M. Rumble.
The following diploma and certificates have been D.I.H.-Mary Jackson.

D.I.H.-Mary Jackson. C.I.H.-A. A. I. Awad, A. W. Baird, R. E.
Dickie, Bik Ann Lau, H. Megerdichian, S. Pump-
ford, M. A. Sadiq. IWith high commendation. 2 With commendation.

ROYAL COLLEGE OF SURGEONS OF FACUlty OF Dental SuRgery

Professor G. L. Slack, London Hospital Medical College Dental School, and Professor B. E. D elected dean and vice-dean, respectively, of the Faculty of Dental Surgery.

ROYAL COLLEGE OF SURGEONS IN IRELAND F.R.C.S.I.-J. Cox, P. W. Eustace, M. Flynn, R. J. Gay, J. S. Hanson, N. M. Heney, V. K. Kapur,
J. R. Nixon, J. A. O'Donnell, L. A. Velia, P. J. Robinson, G. C. Slack, R. Y. Tan, A. Tun.
F.F.A. R.C.S.I.-Y. E. Angole, Barbara F.F.A. R.C.S.I.-Y. E Angole, Barbara J.
Burbidge, P. L. Flynn, Margaret D. Milne, Bani Mondal, S. L. Samuels, H. Z. Su, K. P. Sukumaran.
D.A.-Rosalie H. Auckland, Marie T Burns, D.A.- Rosalie H. Auckland, Marie T Burns,
Christine P. Dawkins, Frances P. M. Donovan, Maureen J. Mchaels, Greta E. M. Opdebeeck Ormon 1, C. H. Seah, S. A. Taha. Bakir, Aubrey de V. Bourke, Kate C. Bryant, Norah Chahim, M. J. C. Conran, M. O. Dalahmeh, B. R. Denham, Sylvia S. Dockeray, G. R. D-nald. Brona S. Donnellan, P. J. Donohoe, D. F. Dr.f,, J. A. Flattery, D. G. Gill, Elizabeth A. Griffin, I. P. Camille De S. Lazaro, D. F. Lillis, P. P. J. Maguire, Kitbia Maieed, Marraret A. Meyer, M. R.

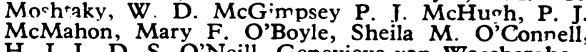
H. T. L. D. S. O'Neill, Genevieve van Waesber-he. D.O.-Anwar-ul-haq, O. S. Awotar, Mary E. C.
Flynn, M. Iabal, Z. M. Jaouni, H. C. Jindal, Rosaline Kelly, H. N. Mandalia, E. Moola, M. Rafi. Orekondy Siddalingeswara, H. M. Singh, S. A.
Ali Wasti, A. A. Zaidi.

\section{CORRECTION}

\section{Liver Damage and Methotrexate}

In a letter by Dr. H. Baker (26 June, p. 776) the plasma levels of methotrexate were wroncly printed. This should have read "Oral doses of $2.5 \mathrm{mg}$, given five days each week for psoriasis, produce peak plasma levels of about $200 \mathrm{ng} / \mathrm{ml}$ and levels above $30 \mathrm{ng} / \mathrm{ml}$ may be obtained for up to five hours daily ....”.

\section{Royal College of Psychiatrists}

In a letter by Dr. A. Shapiro (17 July, p. 188) he was inadvertently designated "Chairman of the Section of Psychiatry." This should have read "Chairman of the Section of Mental Deficiency" of the Royal College of Psychiatrists.

\section{Cerebral Vasodilators}

In the article on "Cerebral Vasodilators" (19 June 1971 , p. 702) buphenine (Dilacol) was described as a cerebral vasodilator. Dilacol contains only $1.5 \mathrm{mg}$ of buphenine hydrochloride which would have little vasodilator effect compared with Perdilatal Forte, which contains $6 \mathrm{mg}$. The sentence should therefore have read "Various other drugs have been used as cerebral vasodilators including nicotinyl tartrate (Ronicol); and buphenine hydrochloride (Perdilatal Forte), which like cyclandelate and isoxsuprine predominantly affects blood vessels in skeletal muscle."

\section{Notice to Authors}

When original articles and letters for publication are not submitted exclusively to the British Medical fournal this must be stated.

Correspondence on editorial business should be addressed to the Editor, British Medical fournal, B.M.A. House, Tavistock Square, London WC1H 9JR. Telephone: 01-387 4499. Telegrams : Aitiology, London, W.C.1.

Authors wanting reprints of their articles should notify the Publishing Manager, B.M.A. House, Tavistock Square, WC1H 9JR, on receipt of proofs. 